Plagiarism and fraud

\section{Plagiarism and fraud}

\section{J Mayberry}

\section{The death knell of research}

A s editor, the appearance of an excellent article is always exciting. The quality that sometimes exudes from every line is a joy to the reader. The thought that your journal has been selected by the authors gives you an inner glow. The citation index will rise, subscriptions increase, friends and colleagues will praise the journal, and other prestigious authors will submit articles. There may be a nagging question as to why you were selected, but for some there will not even be such a doubt.

When the paper deals with new and effective treatments you can expect press interest. Patients will be excited by the possibility that their incurable disease may now be treatable. They have hope. When a paper deals with the origins of disease, the role of environmental and genetic factors can be exposed and preventative programmes developed. The whole purpose of research is confirmed by such work.

What should our response be when the paper is a fraud-when the study never happened, or the results are fabricated or changed? The hopes of patients are dashed, new prevention or treatment plans are pointless and have diverted effort from the true path. We

\title{
Cardiology
}

\section{Cardiology update \\ D Gray}

\section{A new series on cardiovascular disease}

$\mathrm{T}$ his issue of Postgraduate Medical Journal introduces a new series that will review some recent developments, and some emerging technologies, in cardiovascular medicine and how they impact on patient care.

Medical practice has undergone revolutionary changes in the last 20 years, not least in the field of cardiology. Clinical investigators, aided by an eager pharmaceutical industry, have published a wide variety of well designed and conducted clinical trials which have established the benefits of novel and exciting approaches to treatment, resulting in a greatly expanded medical and surgical armamentarium. As a result, patients with the common presentations of cardiac disease, particularly heart failure, acute coronary syndromes and hypertension, have benefited from therapy which has reduced morbidity and mortality, accompanied by significantly improved quality of life.

The electronics' industry has played an important part too, developing new modalities of imaging which have largely replaced invasive tests, particularly in paediatric care; for example, echocardiography achieves more in five minutes with baby on Mum's lap than does cardiac catheterisation of an anaesthetised infant. Magnetic resonance imaging is at an early stage of development but holds the promise of non-invasive visualisation of the coronary arteries.

New diagnostic tests have emerged. For example, the enzyme creatine should all decry such fraud and, as coauthors on papers, ensure to the best of our abilities that we prevent such episodes from occurring.

The duplication of huge chunks of text in an article without referencing its source may not have such direct impact on patient care, but its perpetrators are also guilty of fraud; intent on promoting their own careers and showing little concern for the truth.

Sadly, we see such cases at the Postgraduate Medical Journal and their submission is a disservice to the journal and its readers. Fraud and plagiarism are the death knell of research. They bring individuals, institutions, and academic journals into disrepute. Such authors have stepped beyond the pale of acceptable practice and, once there, can never return to clinical or scientific circles.

\section{Postgrad Med J 2003;79:605}

Correspondence to: Dr John Mayberry, Editor, Postgraduate Medical Journal, Leicester General Hospital, Gwendolen Road, Leicester LE5 4PW, UK; pmj@btinternet.com

kinase and the structural component troponin have displaced non-specific markers such as aspartate aminotransferase, lactate dehydrogenase, and hydroxybutyrate dehydrogenase. This has led to a reappraisal of the diagnosis of acute manifestations of coronary disease, myocardial infarction, and unstable angina and their reclassification as the acute coronary syndromes.

The next 20 years are likely to prove as exciting as the last, with new drugs, new surgical approaches and new lines of research, not least in DNA technology. Enthusiasts, and the next generation of doctors, must wait to see whether the potential of DNA profiling to identify those at risk of coronary and other disease benefits patients and society or proves a bonanza for the insurance world.

This series will cover aspects of basic science, clinical trials, investigation, and treatment of cardiac disease to keep readers up to date with recent and future developments in cardiovascular disease.

Postgrad Med J 2003;79:605

Correspondence to: Dr David Gray, Series Editor, University Hospital, Clifton Boulevard, Nottingham NG7 2UH, UK.

d.gray@nottingham.ac.uk 